\title{
EXOTIC BROWNIAN MOTIONS
}

\author{
Hirofumi OSADA \\ (Received 24 March 2006) \\ Dedicated to Professor Masatoshi Fukushima on his 70th birthday
}

\begin{abstract}
We develop a method to construct diffusions on singular sets. Our method can be applied to various fractal sets including Sierpiński carpets, the Sierpiński gasket and the Menger sponge.
\end{abstract}

\section{Introduction}

A diffusion $(X, \mathrm{P})$ with state space $\mathrm{S}$ is a strong Markov process with continuous sample paths over the life time. Here and throughout $\mathrm{P}=\left\{\mathrm{P}_{x}\right\}_{x \in S}$ denotes the family of diffusion measures and $X=\left\{X_{t}\right\}$ is the canonical S-valued process starting at $x$ under $\mathrm{P}_{x}$ (see $[\mathbf{8}]$ ).

Since the pioneer work of Kolmogorov [10], diffusions have been one of the main subjects in probability theory. So far various methods to construct diffusions have been investigated including Kolmogorov's analytic method, the stochastic differential equation (SDE) approach by Itô, the martingale approach by Stroock-Varadhan and the Dirichlet form approach by Fukushima. All of these methods except the last one require some regularity of the state space in the sense that the state space $S$ has to be a domain of Euclidean spaces or smooth manifolds.

On the other hand, there is increasing interest in singular spaces. Here singular spaces mean closed sets in $\mathbb{R}^{d}$ with no manifold structure even when they are local. Typical examples of such singular spaces are the self-similar fractals such as the Sierpiński gasket, the Sierpiński carpet and the Menger manifolds.

2000 Mathematics Subject Classification: Primary 60J60; Secondary 60J45.

Keywords and Phrases: diffusion; Sierpiński carpet; fractal; Dirichlet form.

This article is an invited contribution to a special issue of the Kyushu Journal of Mathematics commemorating the sixtieth volume. 
The purpose of this paper is to develop a method to construct diffusions on singular spaces by using the Dirichlet form theory.

In the theory of regular Dirichlet forms, the standard Brownian motion is given by the triplet $\left(\mathcal{E}^{d}, H^{1}\left(\mathbb{R}^{d}\right), L^{2}\left(\mathbb{R}^{d}, \lambda^{d}\right)\right)$, where $\lambda^{d}$ is the $d$-dimensional Lebesgue measure and $\mathcal{E}^{d}$ is the bilinear form on $H^{1}\left(\mathbb{R}^{d}\right)$ such that

$$
\mathcal{E}^{d}(f, g)=\int_{\mathbb{R}^{d}} \mathbb{D}[f, g] d \lambda^{d} .
$$

Here $\mathbb{D}$ is the canonical square field defined by

$$
\mathbb{D}[f, g]:=\frac{1}{2} \sum_{i=1}^{d} \frac{\partial f}{\partial x_{i}} \cdot \frac{\partial g}{\partial x_{i}} .
$$

We remark that the measures in the energy form $\mathcal{E}^{d}$ and in the $L^{2}$-space $L^{2}\left(\mathbb{R}^{d}, \lambda^{d}\right)$ are common. So we slightly change the point of view and represent the Brownian motion as a triplet

$$
\left(\mathbb{D}, \lambda^{d}, \mathcal{D}_{0}\right) \text {. }
$$

Here $\mathcal{D}_{0}$ is a subspace of $L^{2}\left(\mathbb{R}^{d}, \lambda^{d}\right)$ such that $\overline{\mathcal{D}}_{0}^{\mathcal{E}}=H^{1}\left(\mathbb{R}^{d}\right)$. There are many choices of $\mathcal{D}_{0}$, say $C_{0}^{n}\left(\mathbb{R}^{d}\right)(n=1,2, \ldots)$.

This interpretation has an advantage because one can replace $\lambda^{d}$ by other measures $\mu$ to produce new diffusions associated with $\left(\mathbb{D}, \mu, \mathcal{D}_{0}\right)$. Indeed, when $\mu$ has a lower semi-continuous density with respect to $\lambda^{d}$, such diffusions are called distorted Brownian motions and have been studied by Fukushima [6, 7]. Now we are naturally led to the question of whether or not another class of Radon measures producing new diffusions would exist. We give an affirmative answer for this question and find a class of Radon measures that yield good diffusions on their topological supports. All of these measures are singular to the Lebesgue measure and the state space of the resulting diffusions can be taken to be singular sets such as the fractals mentioned above.

Let $\mathbb{S}=\{\mathrm{s}\}$ be a countable collection of disjoint $d_{\mathrm{s}}$-dimensional smooth submanifolds in $\mathbb{R}^{d}$, where $d \geq 2$. The dimension $d_{\mathrm{s}}$ of sub-manifold $\mathrm{s}$ may take $1 \leq d_{\mathrm{s}} \leq d-1$, although it holds that $d_{\mathrm{s}}=d-1$ for all $\mathrm{s} \in \mathbb{S}$ in many interesting cases. Let $\mathrm{S}$ be the closed set on $\mathbb{R}^{d}$ given by the closure of $\bigcup_{\mathbf{s} \in \mathbb{S}} \mathrm{s}$ :

$$
S=\overline{\bigcup_{s \in \mathbb{S}} s} \text {. }
$$

Exotic Brownian motions $\mathrm{P}=\left\{\mathrm{P}_{x}\right\}_{x \in \mathrm{S}}$ are diffusions with state space $\mathrm{S}$ given by the Dirichlet form $(\mathcal{E}, \mathcal{D})$ on $L^{2}(\mathrm{~S}, \mu)$, which is the closure of the form $\left(\mathcal{E}, \mathcal{D}_{0}(\mathrm{~S})\right)$. 
Here $\mathcal{D}_{0}(\mathrm{~S})$ is a subspace of $C_{0}(\mathrm{~S})$ (see (2.4) for the definition), and the energy form $\mathcal{E}$ is given by

$$
\mathcal{E}(f, g):=\int_{\mathrm{S}} \mathbb{D}[f, g] d \mu,
$$

with the canonical square field $\mathbb{D}$ in (1.1) and the Radon measure $\mu$ of the form

$$
d \mu=\sum_{\mathbf{s} \in \mathbb{S}} w_{\mathbf{s}} d \lambda_{\mathbf{S}}
$$

where $\lambda_{\mathrm{S}}$ is the $d_{\mathrm{S}}$-dimensional volume on the $d_{\mathrm{S}}$-dimensional sub-manifold $\mathrm{s}$ and $w_{\mathrm{S}}$ are non-negative measurable functions. We take $w_{\mathrm{s}}$ in such a way that $\mu$ is a Radon measure whose topological support is equal to $S$. Later, we suppose a mild condition on $w_{\mathrm{S}}$ for the closability of the form $\left(\mathcal{E}, \mathcal{D}_{0}(\mathrm{~S})\right)$. We remark that this condition is satisfied if, say, $w_{\mathrm{s}}$ is bounded and lower semi-continuous.

In the first main theorem (Theorem 2.1), we prove that $\left(\mathcal{E}, \mathcal{D}_{0}(S)\right)$ is closable on $L^{2}(\mathrm{~S}, \mu)$ and its closure $(\mathcal{E}, \mathcal{D})$ is a strongly local, regular Dirichlet form. Hence, by the Dirichlet form theory, we obtain the symmetric diffusion $\mathrm{P}$ associated with $(\mathcal{E}, \mathcal{D})$ on $L^{2}(\mathrm{~S}, \mu)$.

We emphasize that we will not take $\mathcal{D}_{0}(\mathrm{~S})$ as $C_{0}^{1}(\mathrm{~S})$ as we cannot expect that $\left(\mathcal{E}, C_{0}^{1}(\mathrm{~S})\right)$ is closable on $L^{2}(\mathrm{~S}, \mu)$ because $\mu$ is singular to the $d$-dimensional Lebesgue measure. One of our key points is that we take the domain $\mathcal{D}_{0}(\mathrm{~S})$ very carefully in order to prove the closability of $\left(\mathcal{E}, \mathcal{D}_{0}(\mathrm{~S})\right)$.

In the second main theorem (Theorem 2.2), we prove, under a set of assumptions on isoperimetric inequalities on $\mu$, that these diffusions $\mathrm{P}=\left\{\mathrm{P}_{x}\right\}$ have positive density $p(t, x, y)$ with respect to $\mu$ satisfying the following:

$$
p(t, x, y) \leq c_{1} t^{-\alpha / 2} e^{-c_{2}|x-y|^{2} / t} \quad \text { for all } x, y \in \mathrm{S}, 0<t<\infty,
$$

where $\alpha \geq 1, c_{1}$ and $c_{2}$ are positive constants independent of $(t, x, y)$. The exponent $\alpha$ is different from the original space dimension $d$. Moreover, for any compact set $K \subset(0, \infty) \times \mathrm{S} \times \mathrm{S}$ there exists a positive constant $c_{K}$ such that

$$
c_{K} \leq p(t, x, y) \text { for all }(t, x, y) \in K .
$$

Recall that $S \in \mathbb{S}$ are pairwise disjoint and, by definition, the energy of the diffusion is concentrated on $\sum_{\mathbf{s} \in \mathbb{S}} \mathbf{S}$. The above inequalities nevertheless imply that the diffusion is non-degenerate; it moves about all over $\mathbf{S}$. Even if $\mathbb{S}$ consists of disjoint compact sets, we have many examples that both of (1.5) and (1.6) hold.

The organization of the paper is as follows. We give the setup and main theorems in Section 2. In Section 3 we give some examples. We also introduce exotic Brownian 
motions on $\mathbb{R}^{d}$ and investigate some properties. In Section 4 we prove Theorem 2.1. In Section 5 we prove a co-area formula for the Radon measures in $\mathbb{R}^{d}$ and deduce Sobolev inequalities. In Section 6 we prove Theorem 2.2. In Section 7 we prove Theorems 3.4 and 3.5.

\section{Setup and main results}

Let $\mathbb{S}=\{\mathbf{s}\}$ denote a collection of smooth sub-manifolds in $\mathbb{R}^{d}$. The dimension $d_{\mathbf{S}}$ of $\mathrm{s}$ may depend on $\mathrm{s}$ and ranges over $1 \leq d_{\mathrm{s}} \leq d-1$. We denote by $\overline{\mathrm{s}}$ the closure of $\mathrm{s}$ in $\mathbb{R}^{d}$ and set $\partial \mathbf{s}=\overline{\mathbf{s}} \backslash \mathbf{s}$. We assume $\mathbb{S}=\{\mathbf{s}\}$ satisfying the following assumptions.

(A1) The cardinality of $\mathbb{S}$ is countable.

(A2) For each $\mathrm{s}$ the closure $\overline{\mathrm{s}}$ is compact and there exists a smooth sub-manifold $\tilde{\mathrm{s}}$ satisfying $d_{\mathbf{s}}=d_{\tilde{\mathbf{s}}}$ and $\overline{\mathbf{s}} \subset \tilde{\mathbf{s}}$.

(A3) $\mathrm{s} \cap \mathrm{s}^{\prime}=\emptyset$ for $\mathrm{s}, \mathrm{s}^{\prime} \in \mathbb{S}$ such that $\mathrm{s} \neq \mathrm{s}^{\prime}$.

(A4) $\lambda(\partial \mathbf{s})=0$ for all $\mathbf{s} \in \mathbb{S}$, where $\lambda=\sum_{\mathbf{s} \in \mathbb{S}} \lambda_{\mathbf{s}}$ and $\lambda_{\mathrm{s}}$ denotes the $d_{\mathbf{s}}$-dimensional volume on $\mathrm{s}$.

We remark that assumption (A2) means that each $s$ has a tubular neighborhood. Set $\mathbf{S}=\overline{\bigcup_{\mathbf{s} \in \mathbb{S}} \mathbf{S}}, d \mu=\sum_{\mathbf{s} \in \mathbb{S}} w_{\mathbf{S}} d \lambda_{\mathbf{s}}$ as before. We assume that $\left\{w_{\mathbf{s}}\right\}$ satisfies the following.

(A5) $\mu$ is a Radon measure whose topological support is equal to $\mathrm{S}$.

(A6) For each $\mathrm{s} \in \mathbb{S}$ the form $\left(\mathcal{E}_{\mathrm{s}}, \operatorname{Lip}_{0}\left(\mathbb{R}^{d}\right)\right)$ is closable on $L^{2}\left(\mathbb{R}^{d}, w_{\mathrm{S}} d \lambda_{\mathrm{s}}\right)$.

Here

$$
\mathcal{E}_{\mathrm{S}}(f, g)=\int \frac{1}{2}\left(\nabla_{\mathrm{s}} f\right) \cdot\left(\nabla_{\mathrm{s}} g\right) w_{\mathrm{s}} d \lambda_{\mathrm{s}},
$$

where $\nabla_{\mathrm{S}}$ is the unit tangential derivative on $\mathrm{S}$ and $\operatorname{Lip}_{0}\left(\mathbb{R}^{d}\right)$ is the set consisting of the Lipschitz continuous functions on $\mathbb{R}^{d}$ with compact support. We remark that, by definition, $\nabla_{\mathrm{s}} f$ is a $d_{\mathrm{s}}$-dimensional vector. We naturally regard $\nabla_{\mathrm{s}}$ as an element of $\mathbb{R}^{d}$. So $\cdot$ denotes the standard inner product in $\mathbb{R}^{d}$.

Let $\mathcal{D}_{0}\left(\mathbb{R}^{d}\right)=\left\{f: \mathbb{R}^{d} \rightarrow \mathbb{R} ; f\right.$ satisfies (2.1), (2.2), (2.3) $\}$.

$f$ is Lipschitz continuous with compact support

$$
\|\mathbb{D}[f, f]\|_{L^{\infty}(\lambda)}<\infty
$$

$$
\nabla_{\mathrm{s}, n} f=0 \quad \text { for } \lambda \text {-almost every } x \in \mathrm{s} \quad(\forall \mathrm{s} \in \mathbb{S}) .
$$

Here $\nabla_{\mathrm{s}, n}$ denotes the normal derivative on $\mathrm{s}$. So $\nabla_{\mathrm{s}, n} f(x)$ is a $\left(d-d_{\mathrm{S}}\right)$-dimensional vector. Let

$$
\mathcal{D}_{0}(\mathrm{~S})=\left\{f ; f=\left.g\right|_{\mathrm{S}} \text { for some } g \in \mathcal{D}_{0}\left(\mathbb{R}^{d}\right)\right\}
$$


We remark the 'normal' derivative of $f$ is vanished in the dense subset $\bigcup_{\mathbf{s} \in \mathbb{S}} \mathbf{S}$ of $\mathbf{S}$. In the next theorem we will see that $\mathcal{D}_{0}(\mathrm{~S})$ is nevertheless dense in $C_{0}(\mathrm{~S})$.

We now state one of the main theorems.

THEOREM 2.1. Assume that assumptions $(A 1)-(A 6)$ hold. Then $\left(\mathcal{E}, \mathcal{D}_{0}(\mathrm{~S})\right)$ is closable on $L^{2}(\mathrm{~S}, \mu)$, and its closure $(\mathcal{E}, \mathcal{D})$ is a strongly local, regular Dirichlet form on $L^{2}(\mathrm{~S}, \mu)$.

We refer to [8] for the definition of a strongly local, regular Dirichlet form. Combining Theorem 2.1 with the Dirichlet form theory in [8] we obtain the following.

COROLLARY 2.1. There exists a $\mu$-symmetric diffusion $\mathrm{P}=\{\mathrm{P}\}_{x \in \mathrm{S}}$ on $\mathrm{S}$ associated with $\left(\mathcal{E}, \mathcal{D}, L^{2}(\mathrm{~S}, \mu)\right)$.

The properties of the diffusions such as non-degeneracy, transience and estimates of the transition probability density greatly depend on how the collection of submanifolds $\mathbb{S}=\{\mathbf{s}\}$ is organized and how the weight functions $\left\{w_{\mathrm{s}}\right\}$ are chosen. We give a criterion for the non-degeneracy of diffusion in terms of isoperimetric constants. For this purpose we introduce the surface volume $\sigma_{\mu}$ with respect to $\mu$. For $(d-1)$-dimensional smooth sub-manifolds $S$ with the $\epsilon$-tubular neighborhood $S^{\epsilon}$ for small enough $\epsilon$ we set

$$
\sigma_{\mu}(S)=\liminf _{\epsilon \rightarrow 0} \frac{1}{2 \epsilon} \mu\left(S^{\epsilon}\right) .
$$

We remark that $\sigma_{\mu}$ is super additive, $\sigma_{\mu}\left(S_{1}+S_{2}\right) \geq \sigma_{\mu}\left(S_{1}\right)+\sigma_{\mu}\left(S_{2}\right)$, by definition.

\section{Isoperimetric constant of $\mu$}

Let $\alpha \geq 1$ be a constant and set $\tilde{\alpha}=1-1 / \alpha$. For an open set $O$ we set

$$
\mathfrak{I}_{\mu}^{\alpha}(O)=\inf _{q \in Q(O)} \frac{\sigma_{\mu}(\partial q \cap O)}{\mu(q \cap O)^{\tilde{\alpha}}} .
$$

Here $\partial q$ is the boundary of $q$ in $\mathbb{R}^{d}$ and

$$
Q(O)=\left\{q \subset \mathbb{R}^{d} ; q \text { is a bounded open set, } \partial q \text { is smooth, } 0<\mu(q)<\mu(O) / 2\right\} .
$$

Here, when $\mu(O)=\infty$, the last condition simply means $0<\mu(q)<\infty$. 
Chegeer constant

For an open set $O$ we set

$$
\mathfrak{h}_{\mu}(O)=\inf _{q \in Q(O)} \frac{\sigma_{\mu}(\partial q \cap O)}{\mu(q \cap O)} .
$$

In order to obtain a non-degenerate diffusion, we assume that assumptions (A7) and (A8) below hold. For the upper bound we need the following assumption.

(A7) The measure $\mu$ satisfies $\mu\left(\mathbb{R}^{d}\right)=\infty$ and

$$
\mathfrak{I}_{\mu}^{\alpha}\left(\mathbb{R}^{d}\right)>0 \text { for a constant } \alpha \geq 1 .
$$

For the positivity of the transition density we need the following assumption.

(A8) There exists a sequence $\mathbb{X}=\left\{x_{m}\right\}_{m \in \mathbb{N}}$ satisfying the following. For each $x_{m} \in \mathbb{X}$ there exist a constant $\gamma_{m}>0$, a continuous increasing function $h_{m}$ and a metric $\rho_{m}$ being equivalent to the Euclidean metric such that the triplet $\left(\gamma_{m}, h_{m}, \rho_{m}\right)$ makes the constants $c_{3}$ and $c_{4}$ defined below positive. Here

$$
\begin{aligned}
& c_{3}\left(x_{m}, \gamma_{m}, h_{m}, \rho_{m}\right):=\inf \left\{\mathfrak{I}_{\mu}^{\alpha}\left(\mathcal{O}_{m}(\delta)\right) ; \gamma_{m} / 2<\delta<2 \gamma_{m}\right\}, \\
& c_{4}\left(x_{m}, \gamma_{m}, h_{m}, \rho_{m}\right):=\inf \left\{\mathfrak{h}_{\mu}\left(\mathcal{O}_{m}(\delta)\right) ; \gamma_{m} / 2<\delta<2 \gamma_{m}\right\},
\end{aligned}
$$

where $\mathcal{O}_{m}(\delta)=\left\{x \in \mathbb{R}^{d} ; \bar{\rho}_{m}(x)<\delta\right\}$ and $\bar{\rho}_{m}(x)=h_{m}\left(\rho_{m}\left(x_{m}, x\right)\right)$. In addition, we assume that the triplet $\left(\gamma_{m}, h_{m}, \rho_{m}\right)$ is chosen in such a way that

$$
\mathrm{S} \subset \bigcup_{x_{m} \in \mathbb{X}} \mathcal{O}_{m}\left(\gamma_{m} / 2\right)
$$

and that, for a positive constant $c_{5}$, the function $\bar{\rho}_{m}(x)$ satisfies

$$
c_{5}^{-1} \leq \mathbb{D}\left[\bar{\rho}_{m}, \bar{\rho}_{m}\right](x) \leq c_{5} \quad \text { for } \mu \text {-almost every } x \in \mathcal{O}_{m}\left(2 \gamma_{m}\right) \backslash \mathcal{O}_{m}\left(\gamma_{m} / 2\right) .
$$

THEOREM 2.2. (1) Assume that assumption (A7) holds. Then $\mathrm{P}=\left\{\mathrm{P}_{x}\right\}$ has a version of density $p=p(t, x, y)$ with respect to $\mu$ satisfying the following

$$
p(t, x, y) \leq c_{6} t^{-\alpha / 2} e^{-c_{7}|x-y|^{2} / t}
$$

for all $x, y \in \mathrm{S}, 0<t<\infty$. Here $c_{6}$ and $c_{7}$ are positive constants depending only on $\alpha$ and $\mathfrak{I}_{\mu}^{\alpha}\left(\mathbb{R}^{d}\right)$.

(2) Assume that assumptions (A7) and (A8) hold. Then $\mathrm{P}$ has a version of density $p=p(t, x, y)$ with respect to $\mu$ that has a locally uniform lower bound; for each compact set $K \subset(0, \infty) \times \mathrm{S} \times \mathrm{S}$ there exists a positive constant $c_{8}$ such that

$$
c_{8} \leq p(t, x, y) \quad \text { for all }(t, x, y) \in K
$$


The key point of the proof of Theorem 2.2 is the progression from isoperimetric and Chegeer inequalities to inequalities such as Nash, Sobolev and weighted Poincaré inequalities. To do this we need a kind of co-area formula for singular measures $\mu$. In the following proposition, $\mu$ is an arbitrary Radon measure in $\mathbb{R}^{d}$; so $\mu$ is not necessarily of the form (1.4).

Proposition 2.3. Let $f \in C_{0}^{\infty}\left(\mathbb{R}^{d}\right)$ be a non-constant function. Set $A_{t}=$ $\{x ; f(x)=t\}$. Let $\mu$ be a Radon measure on $\mathbb{R}^{d}$ and $\sigma_{\mu}\left(A_{t}\right)$ be the surface volume of $A_{t}$ with respect to $\mu$. Then the following holds:

$$
\int_{\mathbb{R}} \sigma_{\mu}\left(A_{t}\right) d t \leq \int_{\mathbb{R}^{d}}|\nabla f| \mu(d x) .
$$

Remark 2.1. (1) Since the set $A_{t}$ is a compact smooth submanifold for almost every $t \in[\min f(x), \max f(x)]$, a tubular neighborhood of $A_{t}$ exists. So $\sigma_{\mu}\left(A_{t}\right)$ is well defined for almost every $t$.

(2) If $\mu$ has a smooth density with respect to the $d$-dimensional Lebesgue measure, then Proposition 2.3 is well known. In this case an equality holds in (2.14). In the present situation, only an inequality is expected since $\mu$ is singular to the $d$ dimensional Lebesgue measure. Fortunately this is enough for our purpose.

(3) We do not know whether the above proposition is known or not. So we give a proof of Proposition 2.3 in Section 5.

(4) The argument in the proof is local. So the above proposition is naturally extended to Radon measures on complete Riemannian manifolds.

(5) Let $\sigma_{\mu}^{\circ}(\cdot)=\sum_{\mathrm{s}} \sigma_{\int .} w_{\mathrm{s}} d \lambda_{\mathrm{s}}$. Then clearly $\sigma_{\mu}^{\circ} \leq \sigma_{\mu}$. We remark that $\sigma_{\mu}^{\circ} \neq \sigma_{\mu}$ in general. If we replace $\sigma_{\mu}$ by $\sigma_{\mu}^{\circ}$ in (2.14), then the proof of Proposition 2.3 is easy and was done in [11, Lemma 4.1]. In many interesting cases (see Example 3.3, for example), Cheeger constants and the isoperimetric constant $\mathfrak{I}_{\mu}^{\alpha}\left(\mathbb{R}^{d}\right)$ disappear if we replace $\sigma_{\mu}$ by $\sigma_{\mu}^{\circ}$. So this elaboration on Proposition 2.3 is quite important.

Although the assumptions (A1)-(A6) are quite mild and valid for a wide range of models, the last two assumptions (A7) and (A8) depend on the careful arrangement of $\mu$ and $\mathbb{S}$. To ensure that these hold we need a hard analysis depending on each model, so we only give concrete examples in this paper.

\section{Examples}

In this section we give examples. 
Example 3.1. (Exotic Brownian motions on $\mathbb{R}^{d}(d \geq 2)$ ) Let $d \geq 2$ and $N$ be a natural number such that $N \geq 2$. Let $\mathrm{B}^{n}=N^{-n} \mathrm{~B}$, where

$$
\mathrm{B}=\bigcup_{i=1}^{d}\left\{x=\left(x_{1}, \ldots, x_{d}\right) \in \mathbb{R}^{d} ; x_{i} \in \mathbb{Z}, x_{j} \notin \mathbb{Z} \text { for all } j \neq i\right\} \text {. }
$$

We label the connected components of $\mathrm{B}^{n+1} \backslash \mathrm{B}^{n}$ as $\mathrm{B}^{n+1} \backslash \mathrm{B}^{n}=\left\{\mathrm{s}_{k}^{n}\right\}$. We take $\mathbb{S}$ in assumptions (A1)-(A4) as $\mathbb{S}=\left\{\mathbf{S}_{k}^{n}\right\}$. For a given $\alpha>d$, we take weight functions $w_{k}^{n}$ in (1.4) to be constants such that

$$
w_{k}^{n}=c_{5} N^{-(\alpha-d+1) n} \quad \text { for all } n, k .
$$

Here we take the constant $c_{5}$ such that $\mu\left((0,1)^{d}\right)=1$. Then $\mu=\mu^{[\alpha]}$ is a Radon measure on $\mathbb{R}^{d}$ whose support is equal to $\mathbb{R}^{d}$. The measure $\mu$ satisfies the selfsimilarity in the sense that

$$
\mu(N A)=N^{\alpha} \cdot \mu(A) \text { for all } A \in \mathfrak{B}\left(\mathbb{R}^{d}\right)
$$

and other invariance. We see that $\mu$ clearly satisfies assumptions (A5) and (A6).

Let $\mathbb{C}=\left\{(0,1)^{d}+z ; z \in \mathbb{Z}^{d}\right\}$ and $\mathbb{C}^{n}=\left\{N^{-n} \mathbf{c} ; \mathbf{c} \in \mathbb{C}\right\}$. For $\mathbf{c} \in \mathbb{C}^{n}$ and $\delta>0$, we set $\mathbf{c}(\delta)=\left\{x ;|x-\mathbf{c}|_{\max }<N^{-n} \delta\right\}$, where $|x|_{\max }=\max _{i}\left\{\left|x_{i}\right|\right\}$ for $x=\left(x_{i}\right) \in \mathbb{R}^{d}$. For $\mathbf{c} \in \mathbb{C}^{n}$, let $c_{9}(\mathbf{c})$ and $c_{10}(\mathbf{c})$ be the constants defined by

$$
c_{9}(\mathbf{c})=\inf _{N^{-2}<2 \delta<1} \mathfrak{I}_{\mu}^{\alpha}(\mathbf{c}(\delta)), \quad c_{10}(\mathbf{c})=\inf _{N^{-2}<2 \delta<1} \mathfrak{h}_{\mu}^{\alpha}(\mathbf{c}(\delta)) .
$$

In addition, we set $c_{11}$ and $c_{12}$ by

$$
c_{11}=\inf _{\mathbf{c} \in \bigcup_{n \in \mathbb{N}} \mathbb{C}^{n}} c_{9}(\mathbf{c}), \quad c_{12}=\inf _{\mathbf{c} \in \bigcup_{n \in \mathbb{N}} \mathbb{C}^{n}} c_{10}(\mathbf{c}) .
$$

LEMMA 3.1. We have the following.

(1) $c_{11}>0$ and $c_{12}>0$.

(2) $\mathfrak{I}_{\mu}^{\alpha}\left(\mathbb{R}^{d}\right)>0$.

This lemma follows from the same argument as in [11], so we omit the proof. In [11], the Sierpiński carpets were discussed; the proof is clearly valid for this case.

By Lemma 3.1 we see that $\mu$ satisfies assumptions (A7) and (A8). We thus have the following.

THEOREM 3.2. $\mathbb{S}$ and $\mu$ satisfy assumptions (A1)-(A8). The transition density $p=$ $p(t, x, y)$ of the associated diffusion satisfies the global Gaussian upper bound (2.12) and the locally uniform lower bound (2.13). 
We now observe further details of the transition density. For this let

$$
\begin{gathered}
\mathrm{R}=\mathbb{R}^{d} \backslash\left\{\bigcup_{n \in \mathbb{Z}} \mathrm{B}^{n+1} \backslash \mathrm{B}^{n}\right\} \\
\mathrm{R}_{0}=\left\{x \in \mathbb{R}^{d} ; \text { there exists a sequence }\left\{\mathbf{c}_{k}\right\}\right. \text { such that } \\
\left.\mathbf{c}_{k} \in \mathbb{C}^{n_{k}}, \quad \mathbf{c}_{k+1} \subset \mathbf{c}_{k}, \quad x \in \overline{\mathbf{c}}_{k}, \sup _{k} N^{\alpha n_{k}} \mu\left(\overline{\mathbf{c}}_{k}\right)<\infty\right\} \\
\mathrm{R}_{\alpha}=\left\{x \in \mathbb{R}^{d} ; \text { there exists a sequence }\left\{\mathbf{c}_{n}\right\}\right. \text { such that } \\
\left.\mathbf{c}_{n} \in \mathbb{C}^{n}, \quad x \in \overline{\mathbf{c}}_{n}, \quad \mathbf{c}_{n+1} \subset \mathbf{c}_{n}, \quad \limsup _{n \rightarrow \infty} N^{\alpha n} \mu\left(\overline{\mathbf{c}}_{n}\right)<\infty\right\} .
\end{gathered}
$$

Here $\overline{\mathbf{c}}$ is the closure of $\mathbf{c}$. We remark that the sequence $n_{k}$ in the definition of $\mathrm{R}_{0}$ is increasing because $\mathbf{c}_{k+1} \subset \mathbf{c}_{k}$. A moment reflection tells us the following.

LEMMA 3.3. We have the following.

(1) $R, R_{0}$ and $R_{\alpha}$ are dense in $\mathbb{R}^{d}$ and contains the origin.

(2) $\mathrm{R}=\mathrm{R}_{0}$.

As for the continuity and short time estimates of the transition density we have the following.

THEOREM 3.4. There exists a version of $p(t, x, y)$ denoted by the same symbol satisfying the following.

(1) $\operatorname{Each}(t, x, y) \in(0, \infty) \times \mathrm{R} \times \mathrm{R}$ is a continuity point of $p=p(t, x, y)$.

(2) For each $x \in \mathrm{R}_{\alpha}$ there exists a positive constant $c_{13}=c_{13}(x)$ satisfying

$$
c_{13}^{-1} t^{-\alpha / 2} \leq p(t, x, x) \leq c_{13} t^{-\alpha / 2} \quad \text { for all } 0<t<1 .
$$

The inequality (3.7) means that the short time asymptotic behavior of the transition density is different from the standard Brownian motion in the dense subset $\mathrm{R}_{\alpha}$ in spite of the diffusion being non-degenerate and moving around over $\mathbb{R}^{d}$.

As for long time lower bounds we have the following.

THEOREM 3.5. (1) There exists a positive constant $c_{14}$ such that

$$
c_{14} t^{-\alpha / 2} \leq p(t, 0,0) \quad \text { for all } 0<t<\infty .
$$

(2) For each $x \in \mathbb{R}^{d}$ there exists a positive constant $c_{15}=c_{15}(x)$ such that

$$
c_{15} t^{-\alpha / 2} \leq p(t, x, x) \text { for all } 1 \leq t<\infty .
$$


(3) No positive constants $c_{16}$ and $c_{17}$ satisfy the global Gaussian lower bound:

$$
c_{16} t^{-\alpha / 2} e^{-|x-y|^{2} / c_{17} t} \leq p(t, x, y) \quad \text { for all } 0<t<\infty, x, y \in \mathbb{R}^{d} .
$$

We remark that (2.12) and (3.9) imply that the spectral dimension at infinity is $\alpha$. We refer to [13] for another interesting property of exotic Brownian motions.

We prove Theorems 3.4 and 3.5 in Section 7.

Example 3.2. (Exotic Brownian motions on Sierpiński carpets [11]) Let $\mathrm{S}$ denote the $d$-dimensional Sierpiński carpet and $d_{H}$ the Hausdorff dimension of $\mathrm{S}$. If we replace $N$ by $3, \mathbb{R}^{d}$ by $S$ and $d$ by $d_{H}$ in Example 3.1, then the same conclusion as Example 3.1 holds. In [2, 3] Barlow and Bass constructed Brownian motions on Sierpiński carpets. We remark that the exotic Brownian motions on the carpets are different to the Brownian motions constructed by Barlow and Bass.

Example 3.3. (Exotic Brownian motions on bubbles) Let $d \geq 2$. Let $B_{n}$ denote a closed ball with radius $r_{n}$. Let $\mathrm{S}_{n}$ denote the boundary of $B_{n}$. Assume

$$
\begin{gathered}
B_{n} \cap B_{m}=\emptyset \quad \text { for } n \neq m, \\
\overline{\bigcup_{n \in \mathbb{N}} B_{n}}=\mathbb{R}^{d} .
\end{gathered}
$$

Let us take the weight $w_{n}$ in such a way that $w_{n}=r_{n}$. Then it is not difficult to see that assumptions (A7) and (A8) are satisfied with $\alpha=d$.

\section{Proof of Theorem 2.1}

In this section we prove Theorem 2.1. We start by recalling the definition of a tubular neighborhood. Let $\mathrm{S} \in \mathbb{S}$ be fixed. For $x \in \mathrm{s}$, let $U_{\mathrm{S}}(x)$ denote the set of the unit normal vectors at $x$ with respect to the sub-manifold $\mathrm{s}$. We fix a Cartesian coordinate on $\mathbb{R}^{d}$. So we regard each $U_{\mathrm{S}}(x)$ as a subset of $\left\{x \in \mathbb{R}^{d} ;|x|=1\right\}$. We set

$$
U_{\mathrm{s}}=\bigcup_{x \in \mathrm{S}}\left(x, U_{\mathrm{s}}(x)\right) \text {. }
$$

For $z=(x, u) \in U_{\mathrm{S}}$, where $x \in \mathrm{S}$ and $u \in U_{\mathrm{S}}(x)$, let $F_{z}(t)=x+t u$. Set

$$
\epsilon_{\mathrm{S}}=\sup \left\{s ; F_{z}(t) \neq F_{z^{\prime}}\left(t^{\prime}\right) \text { if } z \neq z^{\prime} \in \mathrm{S} \text { and } 0<t, t^{\prime} \leq s\right\}
$$

By assumption (A2) we see $\epsilon_{\mathrm{S}}>0$. For $0<\epsilon<\epsilon_{\mathrm{S}}$, we set the $\epsilon$-tubular neighborhood of $\mathrm{S}$ by $T_{\mathrm{S}}^{\epsilon}=\left\{F_{z}(t) ; z \in U_{\mathrm{S}}, 0 \leq t<\epsilon\right\}$. We define by the inverse 
of $F_{z}(t)$ the map $\varphi_{\mathrm{S}}: T_{\mathrm{S}}^{\epsilon} \rightarrow U_{\mathrm{S}} \times[0, \epsilon) / \sim$, where $\sim$ is the identification such that $(x, u, 0) \sim\left(x, u^{\prime}, 0\right)$.

For constants $a, b$ such that $0 \leq a<b<\epsilon$, let $\ell_{a, b}:[0, \infty) \rightarrow[0, \infty)$ be a smooth and non-decreasing function such that

$$
\ell_{a, b}(t)= \begin{cases}t & b \leq t \\ 0 & 0 \leq t \leq a .\end{cases}
$$

For any $0<a<b$ and $c_{18}>0$ we can and do take $\ell_{a, b}$ in such a way that $\ell_{a, b}=\ell_{a, b}(t)$ is a smooth function in $(a, b, t)$ and that $\ell_{a, b}^{\prime}=\partial \ell_{a, b} / \partial t$ satisfies

$$
0 \leq \ell_{a, b}^{\prime} \leq \frac{b}{b-a}+c_{18}
$$

Let $0<\epsilon<\epsilon_{\mathrm{s}}$. Let $\mathrm{a}, \mathrm{b}: \overline{\mathrm{s}} \rightarrow[0, \epsilon)$ be smooth functions such that

$$
\begin{gathered}
0<\mathrm{a}(x)<\mathrm{b}(x)<\epsilon \quad \text { for } \lambda \text {-almost every } x \in \mathrm{s}, \\
\mathrm{a}(x)=\mathrm{b}(x)=0 \quad \text { for all } x \in \partial \mathrm{s} .
\end{gathered}
$$

We define $\ell_{\mathrm{S}, \mathrm{a}, \mathrm{b}}: \varphi_{\mathrm{S}}\left(T_{\mathrm{s}}^{\epsilon}\right) \rightarrow \varphi_{\mathrm{S}}\left(T_{\mathrm{s}}^{\epsilon}\right)$ by $\ell_{\mathrm{s}, \mathrm{a}, \mathrm{b}}(x, u, t)=\left(x, u, \ell_{\mathrm{a}(x), \mathrm{b}(x)}(t)\right)$. Let us define $m_{\mathrm{s}, \mathrm{a}, \mathrm{b}}: T_{\mathrm{s}}^{\epsilon} \rightarrow T_{\mathrm{s}}^{\epsilon}$ by

$$
m_{\mathrm{s}, \mathrm{a}, \mathrm{b}}=\varphi_{\mathrm{s}}^{-1} \circ \ell_{\mathrm{s}, \mathrm{a}, \mathrm{b}} \circ \varphi_{\mathrm{s}}
$$

Since $m_{\mathrm{s}, \mathrm{a}, \mathrm{b}}$ is the identity near the boundary of $T_{\mathrm{s}}^{\epsilon}$, we naturally extend it to the transformation on $\mathbb{R}^{d}$ by letting $m_{\mathrm{s}, \mathrm{a}, \mathrm{b}}(x)=x$ for $x \notin T_{\mathrm{s}}^{\epsilon}$. We take $\mathrm{a}$, b in such a way that $m_{\mathrm{s}, \mathrm{a}, \mathrm{b}}(x)$ is smooth in $x \in \mathbb{R}^{d}$. We denote this extension by the same symbol $m_{\mathrm{s}, \mathrm{a}, \mathrm{b}}$. Let $\|\cdot\|_{\text {Lip }}$ denote the Lipschitz norm. Then we easily see

$$
1 \leq\left\|m_{\mathrm{s}, \mathrm{a}, \mathrm{b}}\right\|_{\text {Lip }}<\infty .
$$

The following lemma is an immediate consequence of the definition.

LEMMA 4.1. Let $\|\cdot\|_{\infty}$ denote the uniform norm. Let $f$ be a bounded, Lipschitz continuous function. Then

$$
\begin{gathered}
\left\|f \circ m_{\mathrm{s}, \mathrm{a}, \mathrm{b}}\right\|_{\infty}=\|f\|_{\infty} \\
\left\|f \circ m_{\mathrm{s}, \mathrm{a}, \mathrm{b}}-f\right\|_{\infty} \leq\|f\|_{\mathrm{Lip}}\left(\left\|m_{\mathrm{s}, \mathrm{a}, \mathrm{b}}\right\|_{\mathrm{Lip}}-1\right) \\
\left\|f \circ m_{\mathrm{s}, \mathrm{a}, \mathrm{b}}\right\|_{\mathrm{Lip}} \leq\|f\|_{\mathrm{Lip}}\left\|m_{\mathrm{s}, \mathrm{a}, \mathrm{b}}\right\|_{\mathrm{Lip}} \\
f \circ m_{\mathrm{s}, \mathrm{a}, \mathrm{b}}(x)=f(x) \quad \text { for all } x \in \overline{\mathrm{s}} .
\end{gathered}
$$


We remark that $\nabla_{\mathrm{s}, n}\left\{f \circ m_{\mathrm{s}, \mathrm{a}, \mathrm{b}}\right\}(x)=0$ for all $x \in \mathrm{s}$. Let

$$
\begin{gathered}
M_{\mathrm{S}, x, a}(f)=\sup \left\{\frac{\left|f(x)-f\left(\varphi_{\mathrm{S}}(x, u, t)\right)\right|}{t^{2}} ; u \in U_{\mathrm{S}}(x), 0<t \leq a\right\} \\
M_{\mathrm{S}, \mathrm{a}}(f)=\lambda \text {-ess.sup }\left\{M_{\mathrm{S}, x, \mathrm{a}(x)}(f) ; x \in \mathrm{s}\right\} .
\end{gathered}
$$

We remark that if $M_{\mathrm{S}, \mathrm{a}}(f)<\infty$ for some a, then

$$
\partial_{n, \mathrm{~s}} f(x)=0 \quad \text { for all } x \in \mathrm{s} \text { such that } \mathrm{a}(x)>0 .
$$

We label $\mathbb{S}=\left\{\mathbf{s}_{k}\right\}$. For a function $f$ and a sequence $\left\{\left(\mathrm{a}_{k}, \mathrm{~b}_{k}\right)\right\}$ we define $\left\{f_{k}\right\}$ by $f_{0}=f$ and $f_{k}=f_{k-1} \circ m_{\mathbf{s}_{k}, \mathrm{a}_{k}, \mathrm{~b}_{k}}$.

LEMMA 4.2. Let $f \in C_{0}^{\infty}\left(\mathbb{R}^{d}\right)$. For $c_{19}>0$ there exists a sequence $\left\{\left(\mathrm{a}_{k}, \mathrm{~b}_{k}\right)\right\}$ such that the following hold for all $k$ :

$$
\begin{gathered}
\left\|f_{k}\right\|_{\infty}=\|f\|_{\infty} \\
\left\|f_{k}-f_{k-1}\right\|_{\infty} \leq c_{19} 2^{-k} \\
\left\|f_{k}\right\|_{\text {Lip }} \leq\left\|f_{k-1}\right\|_{\text {Lip }}+c_{19} 2^{-k} \\
\max _{l=1, \ldots, k} M_{\mathrm{s}_{l}, \mathrm{a}_{l}}\left(f_{k}\right) \leq 1-2^{-k} .
\end{gathered}
$$

Proof. Equation (4.14) is immediate from (4.7). By (4.4) and the smoothness of $\ell_{a, b}$ in $(a, b, t)$ we can take $\left(\mathrm{a}_{k}, \mathrm{~b}_{k}\right)$ in such a way that $\left\|m_{\mathrm{s}, \mathrm{a}, \mathrm{b}}\right\|_{\text {Lip }}$ is sufficiently close to 1 . Hence, (4.15) and (4.16) follow from (4.8) and (4.9), respectively. Equation (4.17) follows from (4.10) and by choosing $\mathrm{a}_{k}$ in such a way that $\lim \mathrm{a}_{k}(x)=0$ rapidly enough as $x$ goes to $\bigcup_{j=1}^{k} \partial \mathbf{s}_{j}$. We also used assumption (A4) here.

Proof of Theorem 2.1. We first show that $\mathcal{D}_{0}(\mathrm{~S})$ is dense in $L^{2}(\mathrm{~S}, \mu)$ and $C_{0}(\mathrm{~S})$. For this it is enough to prove that, for any $f \in C_{0}^{\infty}\left(\mathbb{R}^{d}\right)$ and $\epsilon>0$, there exists a $g \in \mathcal{D}_{0}(\mathrm{~S})$ such that $\|f-g\|_{\infty}<\epsilon$. For $f \in C_{0}\left(\mathbb{R}^{d}\right)$ and $c_{19}=\epsilon$, let $f_{k}$ be as in Lemma 4.2. Then $\left\{f_{k}\right\}$ is a $\|\cdot\|_{\infty}$-Cauchy sequence by (4.15). Its limit $g=\lim _{k \rightarrow \infty} f_{k}$ satisfies the following by (4.14) and (4.15):

$$
\|f\|_{\infty}=\|g\|_{\infty}, \quad\|f-g\|_{\infty}<\epsilon .
$$

Moreover, by (4.16) we see

$$
\|g\|_{\text {Lip }} \leq\|f\|_{\text {Lip }}+\epsilon<\infty .
$$

This implies that $g$ satisfies (2.1) and (2.2). We next check (2.3). By (4.17) we see

$$
M_{\mathbf{S}_{l}, \mathrm{a}_{l}}(g) \leq \limsup _{k \rightarrow \infty} M_{\mathbf{S}_{l}, \mathrm{a}_{l}}\left(f_{k}\right) \leq 1 \quad \text { for each } l .
$$

So $M_{\mathrm{S}_{l}, \mathrm{a}_{l}}(g)<\infty$ for all $l$, which implies (2.3). 


\section{Proof and applications of the co-area formula}

\subsection{Proof of Proposition 2.3}

Let $\mu$ be a Radon measure on $\mathbb{R}^{d}$ such that $\mu\left(\mathbb{R}^{d}\right)>0$. Let $I=(0,2)$ and $\bar{I}=[0,2]$. Let $f \in C_{0}\left(\mathbb{R}^{d}\right)$ and set

$$
\mathbf{M}=f^{-1}(I) .
$$

The set $\overline{\mathbf{M}}$ will denote the closure of $\mathbf{M}$. In this section, we assume the following:

$$
\begin{gathered}
f(x) \in \bar{I} \quad \text { for all } x \in \mathbb{R}^{d}, \quad \mu(\overline{\mathbf{M}})=1, \\
\left.\quad f\right|_{\mathbf{M}} \in C_{b}^{\infty}(\mathbf{M}),
\end{gathered}
$$

$\mathbf{M}$ is the set of the regular points of $f$.

Remark 5.1. (1) If $f$ is a non-constant smooth function with compact support, then there exist a countable sequence of functions $\left\{f_{m}\right\}$ in $C_{0}\left(\mathbb{R}^{d}\right)$ satisfying (5.1)-(5.3) and sequences of numbers $\left\{a_{m}\right\},\left\{b_{m}\right\}$ such that $f(x)=\sum_{m} a_{m} f_{m}\left(b_{m} x\right)$ holds for all $x \in \mathbb{R}^{d}$. So, to prove Proposition 2.3, it is enough to show that (2.14) holds for functions $f$ satisfying (5.1)-(5.3) instead of $f \in C_{0}^{\infty}\left(\mathbb{R}^{d}\right)$.

(2) Since $|\nabla f|(x)=0$ for all $x \notin \overline{\mathbf{M}}$, we can and do assume without loss of generality that $\mu\left(\overline{\mathbf{M}}^{c}\right)=0$.

Let $A=f^{-1}(\{1\})$. By (5.2) the vector field $\nabla f /|\nabla f|^{2}$ generates the flow $\Phi_{t}$ $(t \in I)$ on $\mathbf{M}$ such that $\Phi_{1}$ is the identity on $A$. Let $\pi: \mathbf{M} \rightarrow A$ be the projection defined by $\Phi_{f(x)}(\pi(x))=x$.

Let $\mathcal{A}^{k}=\left\{a^{k, l}\right\}_{l}$ be a finite partition consisting of elements of $\mathcal{B}(A)$. Let $\mathcal{A}=$ $\bigcup_{k} \mathcal{A}^{k}$. We take $\mathcal{A}^{k}$ in such a way that $\mathcal{B}(A)=\sigma[\mathcal{A}]$ and that $\mathcal{A}^{k}$ is a subdivision of $\mathcal{A}^{k^{\prime}}$ if $k>k^{\prime}$. We note that $\sharp \mathcal{A}$ is countable. We set

$$
\mu^{f}=\mu \circ f^{-1}, \quad \mu^{f, a}(\cdot)=\mu\left(f^{-1}(\cdot) \cap \pi^{-1}(a)\right), \quad \text { where } a \in \mathcal{A} .
$$

Let $F(t)=\mu^{f}((-\infty, t])$ and $F^{a}(t)=\mu^{f, a}((-\infty, t])$. Then these functions are differentiable for almost every $t$. So we set, for almost every $t$

$$
\mathrm{A}_{t}:=\frac{d F}{d t}(t), \quad \mathrm{A}_{t}^{a}:=\frac{d F^{a}}{d t}(t) .
$$

It is clear that

$$
\int_{B} \mathrm{~A}_{t} d t \leq \mu^{f}(B) \quad \text { for all } B \in \mathcal{B}(\mathbb{R})
$$


Since the cardinality of the set $\mathcal{A}$ is countable, we can take a common exceptional set in (5.4). Let $I_{1}$ be a subset of $I$ such that (5.4) holds for all $t \in I_{1}$ and that $I \backslash I_{1}$ has Lebesgue measure zero.

Remark 5.2. Although $f^{-1}(I)$ is the regular points of $f$, we cannot expect (5.4) to hold for all $t \in I$. This is because $\mu$ is singular to the $d$-dimensional Lebesgue measure on $\mathbb{R}^{d}$ in general.

LEMMA 5.1. (1) Let $I_{m, n}=\left[n / 2^{m},(n+1) / 2^{m}\right)$ and $n(t)=n_{m}(t)$ be the natural number such that $t \in I_{m, n(t)}$. Then, for all $t \in I_{1}$,

$$
\begin{gathered}
\mathrm{A}_{t}=\lim _{m \rightarrow \infty} 2^{m} \mu^{f}\left(I_{m, n(t)}\right) \\
\mathrm{A}_{t}^{a}=\lim _{m \rightarrow \infty} 2^{m} \mu^{f, a}\left(I_{m, n(t)}\right) \quad \text { for all } a \in \mathcal{A} .
\end{gathered}
$$

(2) Let $\mathcal{F}=\sigma[f]$. Let $\mu_{t}(\cdot)=\mu(\cdot \mid \mathcal{F})(t)$ denote the regular conditional probability evaluated at $f=t$. Then there exists $I_{2} \subset I$ such that $\mu^{f}\left(I \backslash I_{2}\right)=0$, such that the set $I \backslash I_{2}$ has Lebesgue measure zero and such that, for all $t \in I_{2}$,

$$
\mu_{t}\left(\pi^{-1}(a)\right) \mathrm{A}_{t}=\lim _{m \rightarrow \infty} 2^{m} \mu^{f, a}\left(I_{m, n(t)}\right) \quad \text { for all } a \in \mathcal{A} .
$$

(3) For $a \in \mathcal{A}$, let $a_{t, m}=\pi^{-1}(a) \cap\left\{x \in \mathbb{R}^{d} ;|f(x)-t| \leq 2^{-m-1}\right\}$. Then for all $t \in I_{1} \cap I_{2}$ the following holds:

$$
\mu_{t}\left(\pi^{-1}(a)\right) \mathrm{A}_{t}=\lim _{m \rightarrow \infty} 2^{m} \mu\left(a_{t, m}\right) \quad \text { for all } a \in \mathcal{A} .
$$

Proof. Claim (1) is clear from Lebesgue's density theorem and the fact that the cardinality of $\mathcal{A}$ is countable.

We next prove (2). Let $\mathcal{F}_{m}=\sigma\left[f^{-1}\left(I_{m, n}\right) ; n \in \mathbb{Z}\right]$. Then

$$
\begin{aligned}
\mu_{t}\left(\pi^{-1}(a)\right) & =\mu\left(\pi^{-1}(a) \mid \mathcal{F}\right)(t) \\
& =\lim _{m \rightarrow \infty} \mu\left(\pi^{-1}(a) \mid \mathcal{F}_{m}\right)(t) \quad \text { for } \mu^{f} \text {-almost every } t \\
& =\lim _{m \rightarrow \infty} \frac{2^{m} \mu\left(\pi^{-1}(a) \cap\left\{f \in I_{m, n(t)}\right\}\right)}{2^{m} \mu\left(\pi^{-1}(A) \cap\left\{f \in I_{m, n(t)}\right\}\right)} \\
& =\lim _{m \rightarrow \infty} \frac{2^{m} \mu^{f, a}\left(I_{m, n(t)}\right)}{2^{m} \mu^{f}\left(I_{m, n(t)}\right)} .
\end{aligned}
$$

Here for the second line we used the martingale convergence theorem and $\mathcal{F}=$ $\sigma\left[\mathcal{F}_{m} ; m \in \mathbb{N}\right]$. The third and forth lines are clear by definition. Combining the above equality with (5.7) yields (2).

Claim (3) follows from (2), (5.4) and (5.7). 
Recall that $\left\{\Phi_{t}\right\}$ is a flow on $\mathbf{M}$ and $\mathcal{A}^{k}=\left\{a^{k, l}\right\}$ are partitions of $A$. Let $A_{t}=$ $f^{-1}(\{t\})$. Then $A_{1}=A$ by definition. Let $\left\{a_{t}^{k, l}\right\}_{l}(k \in \mathbb{N})$ denote finite partitions of $A_{t}$ generated by $\left\{\Phi_{t}\right\}$ and $\left\{a^{k, l}\right\}_{l}$; that is,

$$
a_{t}^{k, l}=\Phi_{t}\left(a^{k, l}\right) .
$$

By using (5.2) and (5.3), we can and do take $a^{k, l} \in \mathcal{A}^{k}$ as follows:

$$
\lim _{k \rightarrow \infty} \max _{l} \operatorname{diam} a_{t}^{k, l}=0 \quad \text { for all } t \in I .
$$

Let

$$
\mathrm{M}_{t}^{k, l}=\max _{x \in a_{t}^{k, l}}|\nabla f|(x), \quad \mathrm{m}_{t}^{k, l}=\min _{x \in a_{t}^{k, l}}|\nabla f|(x) .
$$

We set

$$
\mathbf{m}_{t}^{k}=\max _{l}\left\{\mathrm{M}_{t}^{k, l}-\mathrm{m}_{t}^{k, l}\right\} .
$$

Then (5.10) combined with (5.2) implies

$$
\lim _{k \rightarrow \infty} \mathbf{m}_{t}^{k}=0 \quad \text { for all } t \in I .
$$

Lemma 5.2. Let $I_{t, m}=\left[t-2^{-m-1}, t+2^{-m-1}\right]$. For $a^{k, l} \in \mathcal{A}^{k}$ let

$$
a_{t, m}^{k, l}=\pi^{-1}\left(a^{k, l}\right) \cap f^{-1}\left(I_{t, m}\right) .
$$

Let $\Gamma_{t, m}^{k, l}$ denote the $2^{-m-1}$-tubular neighborhood of $a_{t}^{k, l}$. Then there exist increasing sequences $\left\{p_{m}\right\}$ and $\left\{q_{m}\right\}$ of natural numbers, depending only on $t \in I$ and $a^{k, l} \in \mathcal{A}^{k}$, satisfying the following:

$$
\begin{gathered}
a_{t, p_{m}}^{k, l} \subset \Gamma_{t, m}^{k, l} \subset a_{t, q_{m}}^{k, l}, \\
\lim _{m \rightarrow \infty} p_{m}=\lim _{m \rightarrow \infty} q_{m}=\infty, \\
\mathrm{m}_{t}^{k, l} \leq \liminf _{m \rightarrow \infty} 2^{m-p_{m}}, \quad \limsup _{m \rightarrow \infty} 2^{m-q_{m}} \leq \mathrm{M}_{t}^{k, l} .
\end{gathered}
$$

We remark that $a_{t}^{k, l} \subset a_{t, m}^{k, l}$ because of $a_{t}^{k, l}=\pi^{-1}\left(a^{k, l}\right) \cap f^{-1}(\{t\})$, and that (5.14) implies $p_{m} \geq q_{m}$.

Proof. Since $a_{t}^{k, l} \subset A_{t} \subset \mathbf{M}$ for all $t \in I$ and $A_{t}$ is a compact set, Lemma 5.2 follows from (5.2) and (5.3). 
LEMMA 5.3. For all $t \in I_{1} \cap I_{2}, a^{k, l} \in \mathcal{A}$ and $k$ the following holds:

$$
\begin{gathered}
\mathrm{m}_{t}^{k, l} \mu_{t}\left(a_{t}^{k, l}\right) \mathrm{A}_{t} \leq \sigma_{\mu}\left(a_{t}^{k, l}\right) \leq \mathrm{M}_{t}^{k, l} \mu_{t}\left(a_{t}^{k, l}\right) \mathrm{A}_{t}, \\
\left\{\sum_{l} \mathrm{~m}_{t}^{k, l} \mu_{t}\left(a_{t}^{k, l}\right)\right\} \mathrm{A}_{t} \leq \sigma_{\mu}\left(A_{t}\right) \leq\left\{\sum_{l} \mathrm{M}_{t}^{k, l} \mu_{t}\left(a_{t}^{k, l}\right)\right\} \mathrm{A}_{t}, \\
\sigma_{\mu}\left(A_{t}\right)=\sum_{l} \sigma_{\mu}\left(a_{t}^{k, l}\right) .
\end{gathered}
$$

Proof. By (5.14) we have

$$
2^{m-p_{m}} 2^{p_{m}} \mu\left(a_{t, p_{m}}^{k, l}\right) \leq 2^{m} \mu\left(\Gamma_{t, m}^{k, l}\right) \leq 2^{m-q_{m}} 2^{q_{m}} \mu\left(a_{t, q_{m}}^{k, l}\right) .
$$

By (5.15) we can apply (5.9) to see

$$
\lim _{m \rightarrow \infty} 2^{p_{m}} \mu\left(a_{t, p_{m}}^{k, l}\right)=\lim _{m \rightarrow \infty} 2^{q_{m}} \mu\left(a_{t, q_{m}}^{k, l}\right)=\mu_{t}\left(a_{t}^{k, l}\right) \mathrm{A}_{t} .
$$

Hence, by taking the limit infimum in (5.20) and by using (5.16)

$$
\mathrm{m}_{t}^{k, l} \mu_{t}\left(a_{t}^{k, l}\right) \mathrm{A}_{t} \leq \liminf _{m \rightarrow \infty} 2^{m} \mu\left(\Gamma_{t, m}^{k, l}\right) \leq \mathrm{M}_{t}^{k, l} \mu_{t}\left(a_{t}^{k, l}\right) \mathrm{A}_{t}
$$

Since $\liminf _{m \rightarrow \infty} 2^{m} \mu\left(\Gamma_{t, m}^{k, l}\right)=\sigma_{\mu}\left(a_{t}^{k, l}\right)$ by definition, we obtain (5.17).

The proof of (5.18) is similar to that of (5.17), so we omit it.

By summing over $l$ in (5.17) we obtain

$$
\left\{\sum_{l} \mathrm{~m}_{t}^{k, l} \mu_{t}\left(a_{t}^{k, l}\right)\right\} \mathrm{A}_{t} \leq \sum_{l} \sigma_{\mu}\left(a_{t}^{k, l}\right) \leq\left\{\sum_{l} \mathrm{M}_{t}^{k, l} \mu_{t}\left(a_{t}^{k, l}\right)\right\} \mathrm{A}_{t} .
$$

Hence, by combining (5.18) and (5.21) with (5.12) we have

$$
\begin{aligned}
\left|\sigma_{\mu}\left(A_{t}\right)-\sum_{l} \sigma_{\mu}\left(a_{t}^{k, l}\right)\right| & \leq \sum_{l}\left\{\mathbf{M}_{t}^{k, l}-\mathrm{m}_{t}^{k, l}\right\} \mu_{t}\left(a_{t}^{k, l}\right) \mathbf{A}_{t} \\
& \leq \mathbf{m}_{t}^{k} \mu_{t}\left(A_{t}\right) \mathbf{A}_{t} .
\end{aligned}
$$

Then (5.19) follows from (5.13), immediately.

Combining Lemmas 5.1 and 5.2 we obtain the following.

LEMMA 5.4. For all $t \in I_{1} \cap I_{2}$ the following holds:

$$
\sigma_{\mu}\left(A_{t}\right)=\int_{A_{t}}|\nabla f| \mu_{t}(d x) \mathbf{A}_{t}
$$


Proof. By using (5.11) and (5.17) we have, for any choice of points $x_{t}^{k, l} \in a_{t}^{k, l}$,

$$
\left|\sigma_{\mu}\left(a_{t}^{k, l}\right)-\right| \nabla f\left|\left(x_{t}^{k, l}\right) \mu_{t}\left(a_{t}^{k, l}\right) \mathrm{A}_{t}\right| \leq\left\{\mathrm{M}_{t}^{k, l}-\mathrm{m}_{t}^{k, l}\right\} \mu_{t}\left(a_{t}^{k, l}\right) \mathrm{A}_{t} .
$$

Recall that $\sigma_{\mu}\left(A_{t}\right)=\sum_{l} \sigma_{\mu}\left(a_{t}^{k, l}\right)$ by (5.19). Then

$$
\begin{aligned}
\left|\sigma_{\mu}\left(A_{t}\right)-\sum_{l}\right| \nabla f\left|\left(x_{t}^{k, l}\right) \mu_{t}\left(a_{t}^{k, l}\right) \mathbf{A}_{t}\right| & \leq \sum_{l}\left|\sigma_{\mu}\left(a_{t}^{k, l}\right)-\right| \nabla f\left|\left(x_{t}^{k, l}\right) \mu_{t}\left(a_{t}^{k, l}\right) \mathbf{A}_{t}\right| \\
& \leq \mathbf{m}_{t}^{k} \sum_{l} \mu_{t}\left(a_{t}^{k, l}\right) \mathbf{A}_{t} \quad \text { by (5.23) and (5.12) } \\
& =\mathbf{m}_{t}^{k} \mu_{t}\left(A_{t}\right) \mathbf{A}_{t} .
\end{aligned}
$$

Since $\lim _{k \rightarrow \infty} \mathbf{m}_{t}^{k}=0$, we deduce from (5.24) that

$$
\sigma_{\mu}\left(A_{t}\right)=\lim _{k \rightarrow \infty}\left\{\sum_{l}|\nabla f|\left(x_{t}^{k, l}\right) \mu_{t}\left(a_{t}^{k, l}\right)\right\} \mathrm{A}_{t}=\int_{A_{t}}|\nabla f| \mu_{t}(d x) \mathbf{A}_{t} .
$$

Here we used (5.10) for the second equality. We thus completes the proof.

We are now ready to prove Proposition 2.3.

Proof of Proposition 2.3. Recall that $\mu_{t}=\mu(d x \mid f=t)$.

$$
\begin{aligned}
\int_{\mathbb{R}^{d}}|\nabla f|(x) d \mu & =\int_{\mathbb{R}} \int_{A_{t}}|\nabla f|(x) \mu_{t}(d x) \mu^{f}(d t) \quad \text { by disintegration } \\
& \geq \int_{I_{1} \cap I_{2}} \int_{A_{t}}|\nabla f| \mu_{t}(d x) \mathrm{A}_{t} d t \quad \text { by }(5.5) \\
& =\int_{I_{1} \cap I_{2}} \sigma_{\mu}\left(A_{t}\right) d t \quad \text { by Lemma } 5.4 \\
& =\int_{\mathbb{R}} \sigma_{\mu}\left(A_{t}\right) d t
\end{aligned}
$$

Here, for the last line, we used the fact that $\sigma_{\mu}\left(A_{t}\right)=0$ for all $t \notin \bar{I}=[0,2]$ and that $\bar{I} \backslash\left\{I_{1} \cap I_{2}\right\}$ has Lebesgue measure zero.

\subsection{Consequences of the co-area formula}

In this section we collect some consequences of the co-area formula Proposition 2.3. All of such derivations are done by standard arguments. For the reader's convenience we give brief outlines or suitable references for proofs. These results will be used in the next section. 
LEMmA 5.5. (1) Let $\mathcal{O} \subset \mathbb{R}^{d}$ be an open set. Then

$$
\mathfrak{I}_{\mu}^{\alpha}(\mathcal{O}) \cdot\|f\|_{L^{\alpha /(\alpha-1)}(\mathcal{O}, \mu)} \leq \int_{\mathcal{O}}|\nabla f| d \mu
$$

for all $f \in C_{0}^{\infty}\left(\mathbb{R}^{d}\right)$ such that $\mu(\{x ; f(x) \neq 0\} \cap \mathcal{O}) \leq \mu(\mathcal{O}) / 2$.

(2) Let $\mathcal{O} \subset \mathbb{R}^{d}$ be an open set such that $\mu(\mathcal{O})<\infty$. Then

$$
\frac{\mathfrak{h}_{\mu}(\mathcal{O})}{2}\left\|f-\bar{f}_{\mathcal{O}}\right\|_{L^{2}(\mathcal{O}, \mu)} \leq\left\{\int_{\mathcal{O}}|\nabla f|^{2} d \mu\right\}^{1 / 2}
$$

for all $f \in C_{0}^{\infty}\left(\mathbb{R}^{d}\right)$, where $\bar{f}_{\mathcal{O}}=\mu(\mathcal{O})^{-1} \int_{\mathcal{O}} f d \mu$.

Proof. This lemma is well known when $\mu$ is the Lebesgue measure or Riemannian volume, and is derived from the co-area formula Proposition 2.2 in exactly the same fashion, so we omit the proof. See, for example, [5, pp. 97-98] for (5.25), and [5, p. 109, Theorem IV.5.11] for (5.26).

For $\mu$ and $1<\alpha<\infty$, we denote by $c_{20}(\mu, \alpha)$ the constant given by

$$
c_{20}(\mu, \alpha)=\left\{\mathfrak{I}_{\mu}^{\alpha}(\mathcal{O})\right\}^{-1} c_{21}(\alpha),
$$

where $c_{21}(\alpha)$ is such that

$$
c_{21}(\alpha)= \begin{cases}2(\alpha-1) /(\alpha-2) & \text { for } 2<\alpha, \\ 3 / \sqrt{2} & \text { for } 1<\alpha \leq 2 .\end{cases}
$$

LEMMA 5.6. We have the following.

(1) $\quad L^{2}$-Sobolev inequality. Let $\mathcal{O} \subset \mathbb{R}^{d}$ and $f \in C_{0}^{\infty}\left(\mathbb{R}^{d}\right)$ be as in Lemma 5.5(1). Suppose $2<\alpha<\infty$. Then

$$
\|f\|_{L^{2 \alpha /(\alpha-2)}(\mathcal{O}, \mu)} \leq c_{20}(\mu, \alpha)\|\nabla f\|_{L^{2}(\mathcal{O}, \mu)} .
$$

(2) Nash inequality. Suppose $1<\alpha<\infty$. Then

$$
\|f\|_{L^{2}(\mathcal{O}, \mu)}^{1+2 / \alpha} \leq c_{20}(\mu, \alpha)\|f\|_{L^{1}(\mathcal{O}, \mu)}^{2 / \alpha}\|\nabla f\|_{L^{2}(\mathcal{O}, \mu)} .
$$

Proof. By applying Lemma 5.5(1) to $g=|f|^{2(\alpha-1) /(\alpha-2)}$ we obtain (1).

We now prove (2). Suppose $2<\alpha<\infty$. Then, by applying Hölder's inequality to $|f|=|f|^{(2 \alpha+4) /(\alpha+2)}|f|^{2 /(\alpha+2)}$, we see

$$
\|f\|_{L^{2}(\mathcal{O}, \mu)} \leq\|f\|_{L^{2 \alpha /(\alpha-2)}(\mathcal{O}, \mu)}^{\alpha /(\alpha+2)}\|f\|_{L^{1}(\mathcal{O}, \mu)}^{2 /(\alpha+2)} .
$$


Combining this with (5.28) we obtain (5.29) for $2<\alpha<\infty$. Next suppose $1<\alpha \leq 2$. By applying Hölder's inequality to $|f|^{3 \alpha /(\alpha+2)}|f|^{(4-\alpha) /(\alpha+2)}$ we have

$$
\|f\|_{L^{2}(\mathcal{O}, \mu)}^{2} \leq\|f\|_{L^{3 \alpha / 2(\alpha-1)}(\mathcal{O}, \mu)}^{3 \alpha /(\alpha+2)}\|f\|_{L^{1}(\mathcal{O}, \mu)}^{(4-\alpha) /(\alpha+2)} .
$$

Applying (5.25) to $g=|f|^{3 / 2}$ yields

$$
\|f\|_{L^{3 \alpha / 2(\alpha-1)}(\mathcal{O}, \mu)}^{3} \leq \mathfrak{I}_{\mu}^{\alpha}(\mathcal{O})^{-2}\left(\frac{9}{2}\right)\|\nabla f\|_{L^{2}(\mathcal{O}, \mu)}^{2} .
$$

Combining (5.30) and (5.31) yields (5.29) for $1<\alpha \leq 2$.

We next deduce a local $L^{2}$-Sobolev inequality on $\mathcal{O}$ from (5.25) when the volume $\mu(\mathcal{O})$ is finite. So, for $\alpha>1$, we set

$$
\mathbf{a}=\left\{\begin{array}{ll}
\alpha & \text { if } \alpha>2, \\
\alpha+1 & \text { if } \alpha \leq 2,
\end{array} \quad \mathbf{b}=1+2 / \mathbf{a}, \quad \mathbf{c}=2 \mathbf{a} /(\mathbf{a}-2) .\right.
$$

LEMMA 5.7. (Local $L^{2}$-Sobolev inequalities) Let $\mathcal{O} \subset \mathbb{R}^{d}$ be an open set such that $\mu(\mathcal{O})<\infty$. Then

$$
\begin{aligned}
\|f\|_{L^{2 \mathbf{b}}(\mathcal{O}, \mu)} \leq & \left\{\left(c_{20}(\mu, \mathbf{a})\|\nabla f\|_{L^{2}(\mathcal{O}, \mu)}\right)^{1 / \mathbf{b}}\right. \\
& \left.+2\left(\left(\frac{2}{\mu(\mathcal{O})}\right)^{1 / \mathbf{a}}\|f\|_{L^{2}(\mathcal{O}, \mu)}\right)^{1 / \mathbf{b}}\right\}\|f\|_{L^{2}(\mathcal{O}, \mu)}^{1-1 / \mathbf{b}}
\end{aligned}
$$

for all $f \in C_{0}^{\infty}\left(\mathbb{R}^{d}\right)$.

Since the proof is long, we leave it to Appendix A.

Lemma 5.8. Suppose $1<\alpha \leq 2$ and $\mu(\mathcal{O})<\infty$. Then

$$
\mathfrak{I}_{\mu}^{\mathbf{a}}(\mathcal{O}) \geq\left(\frac{2}{\mu(\mathcal{O})}\right)^{1 / \alpha(\alpha+1)} \cdot \mathfrak{I}_{\mu}^{\alpha}(\mathcal{O}) .
$$

Proof. Since

$$
\frac{\alpha}{\alpha+1}=\frac{1}{\alpha(\alpha+1)}+\frac{\alpha-1}{\alpha},
$$

we have, by (2.6) and (2.7),

$$
\mathfrak{I}_{\mu}^{\alpha+1}=\inf _{q \in Q(O)}\left\{\frac{\sigma_{\mu}(\partial q \cap \mathcal{O})}{\mu(q \cap \mathcal{O})^{1 / \alpha(\alpha+1)+(\alpha-1) / \alpha}}\right\} \geq\left(\frac{2}{\mu(\mathcal{O})}\right)^{1 / \alpha(\alpha+1)} \cdot \mathfrak{I}_{\mu}^{\alpha}(\mathcal{O}) .
$$

This completes the proof because $\mathbf{a}=\alpha+1$ by definition. 


\section{Proof of Theorem 2.2}

In this section we prove Theorem 2.2. Let $\mathcal{O}_{m}(\delta)$ and $\gamma_{m}$ be as in (A8) and set $\mu_{m, 1}=\mu\left(\mathcal{O}_{m}\left(\gamma_{m}\right)\right)$ and $\mu_{m, 2}=\mu\left(\mathcal{O}_{m}\left(2 \gamma_{m}\right)\right)$.

LEMMA 6.1. We have the following.

(1) Nash inequality. Assume that assumption (A7) holds. Then there exists a constant $c_{22}$ depending only on $\alpha$ and $\mathfrak{I}_{\mu}^{\alpha}\left(\mathbb{R}^{d}\right)$ such that

$$
\|f\|_{L^{2}\left(\mathbb{R}^{d}, \mu\right)}^{2+4 / \alpha}\|f\|_{L^{1}\left(\mathbb{R}^{d}, \mu\right)}^{-4 / \alpha} \leq c_{22}\|\nabla f\|_{L^{2}\left(\mathbb{R}^{d}, \mu\right)}^{2} \quad \text { for all } f \in C_{0}^{\infty}\left(\mathbb{R}^{d}\right) .
$$

(2) Local Sobolev inequality. Assume that assumption (A8) holds. Let $\mathbf{b}$ be as in (5.32). Then there exists a positive constant $c_{23}=c_{23}\left(x_{m}\right)$ depending only on $c_{5}, \alpha$ and $c_{3}\left(x_{m}\right)$ such that

$$
\begin{aligned}
\|f\|_{L^{2 \mathbf{b}}\left(\mathcal{O}_{m}(\delta), \mu\right)}^{2 \mathbf{b}} \leq & c_{23}\|f\|_{L^{2}\left(\mathcal{O}_{m}(\delta), \mu\right)}^{2(\mathbf{b}-1)} \cdot\left\{\mu\left(\mathcal{O}_{m}(\delta)\right)^{-2 / \alpha}\|f\|_{L^{2}\left(\mathcal{O}_{m}(\delta), \mu\right)}^{2}\right. \\
& \left.+\|\nabla f\|_{L^{2}\left(\mathcal{O}_{m}(\delta), \mu\right)}^{2}\right\}
\end{aligned}
$$

for all $f \in C_{0}^{\infty}\left(\mathbb{R}^{d}\right), \delta \in\left(\gamma_{m} / 2,2 \gamma_{m}\right)$.

(3) Weighted Poincaré inequality. Assume that assumption (A8) holds. Then there exists a positive constant $c_{24}=c_{24}\left(x_{m}\right)$, depending only on $c_{5}, \alpha, \mu_{m, 2} / \mu_{m, 1}$ and $c_{4}\left(x_{m}\right)$, such that

$$
\|f-\bar{f}\|_{L^{2}\left(\mathrm{~S}, \psi_{m}^{2} \mu\right)}^{2} \leq c_{24} \gamma_{m}^{2}\|\nabla f\|_{L^{2}\left(\mathrm{~S}, \psi_{m}^{2} \mu\right)}^{2} .
$$

Here $\bar{f}=\left\{\int_{S} \psi_{m}^{2} d \mu\right\}^{-1} \int_{S} f \psi_{m}^{2} d \mu$ and $\psi_{m}(x)=\bar{\psi}_{m}\left(\bar{\rho}_{m}(x)\right)$, where $\bar{\psi}_{m}$ is the function defined by

$$
\bar{\psi}_{m}(t)= \begin{cases}1 & \text { for } 0 \leq t \leq \gamma_{m}, \\ -\gamma_{m}^{-1} t+2 & \text { for } \gamma_{m} \leq t \leq 2 \gamma_{m}, \\ 0 & \text { for } 2 \gamma_{m} \leq t<\infty .\end{cases}
$$

Proof. Part (1) follows from Lemma 5.6(2). Part (2) follows from Lemma 5.7 and Lemma 5.8. Part (3) follows from Lemma 5.5(2).

By using a mollifier we derive the following from Lemma 6.1 .

LEMMA 6.2. Equations (6.1)-(6.3) also hold for all $f \in \mathcal{D}$.

Combining Lemma 6.2 with [12, Theorem 2.1] we have the following. 
LEMMA 6.3. (Osada [12]) Let $u(t, x)=\int p(t, x, y) \varphi(y) \mu(d y)$, where $\varphi \in$ $L^{2}\left(\mathbb{R}^{d}, \mu\right)$ such that $\varphi \geq 0$. Let $I_{m,-}$ and $I_{m,+}$ be closed intervals in $(0, \infty)$ such that $I_{m,-}=\gamma_{m}^{2}\left[\frac{1}{4}, \frac{3}{4}\right]+T_{m}$ and $I_{m,+}=\gamma_{m}^{2}\left[\frac{5}{4}, 2\right]+T_{m}$ for some $T_{m} \in(0, \infty)$. Assume (A8). Then there exists a positive constant $c_{25}$, depending only on $\alpha, c_{5}$, $c_{3}\left(x_{m}\right), c_{4}\left(x_{m}\right), \mu_{m, 2} / \mu_{m, 1}$ and $\gamma_{m} \mu_{m, 1}^{-1 / \alpha}$ such that (a version of) $u(t, x)$ satisfies

$$
\sup _{I_{m,-} \times \mathcal{O}_{m}\left(\gamma_{m} / 2\right)} u(t, x) \leq c_{25} \inf _{I_{m,+} \times \mathcal{O}_{m}\left(\gamma_{m} / 2\right)} u(t, x) .
$$

Proof of Theorem 2.2. Theorem 2.2(1) follows from Lemma 6.1(1), Lemma 6.2 and Davies' method [4]. Theorem 2.2(2) follows from Theorem 2.2(1) and Lemma 6.3.

\section{Exotic Brownian motions on $\mathbb{R}^{d}$}

In this section we investigate Example 3.1 and prove Theorems 3.4 and 3.5. So let $\mu=\mu^{[\alpha]}$ and $\mathbf{S}=\mathbb{R}^{d}(d \geq 2)$ as in Example 3.1. The parameter $\alpha$ ranges over $d<\alpha<\infty$.

LEMMA 7.1. Let $u(t, x)=\int_{\mathbb{R}^{d}} p(t, x, y) \varphi(y) \mu(d y)$, where $\varphi \geq 0$ and $\varphi \in$ $L^{2}\left(\mathbb{R}^{d}, \mu\right)$. Then each $(t, x) \in(0, \infty) \times \mathrm{R}$ is a continuity point of $u$.

Proof. The proof follows from Lemma 6.3 and the definition of $\mathrm{R}_{0}$ by using the standard argument (see [1, p. 111]). For the reader's convenience we give the proof here.

For $x \in \mathrm{R}_{0}$, let $\left\{\mathbf{c}_{k}\right\}$ be the sequence in (3.5), that is, $\left\{\mathbf{c}_{k}\right\}$ satisfies $\mathbf{c}_{k} \in \mathbb{C}^{n_{k}}$, $\mathbf{c}_{k+1} \subset \mathbf{c}_{k}, x \in \overline{\mathbf{c}}_{k}$ and $\sup _{k} N^{\alpha n_{k}} \mu\left(\overline{\mathbf{c}}_{k}\right)<\infty$. Let $I_{k,-}=N^{-4 n_{k}}\left[\frac{1}{4}, \frac{3}{4}\right]+T_{k}$ and $I_{k,+}=N^{-4 n_{k}}\left[\frac{5}{4}, 2\right]+T_{k}$ such that

$$
I_{k,-} \cup I_{k,+} \subset I_{k-1,+} \subset(0, \infty), \quad t \in I_{k,+}^{\text {int }} \quad \text { for all } k .
$$

Let

$$
m_{k, \pm}=\min _{I_{k, \pm} \times \mathbf{c}_{k}} u(s, y), \quad M_{k, \pm}=\max _{I_{k, \pm} \times \mathbf{c}_{k}} u(s, y) .
$$

We apply Lemma 6.3 with $\gamma_{m}=N^{-2 n_{k}}, I_{m, \pm} \times \mathcal{O}_{m}\left(\gamma_{m} / 2\right)=I_{k, \pm} \times \mathbf{c}_{k}\left(N^{-2} / 2\right)$ to the solutions $M_{k-1,+}-u$ and $u-m_{k-1,+}$. We remark that these are positive solutions because $I_{k,-} \cup I_{k,+} \subset I_{k-1,+}$. Then we obtain, by Lemma 6.3,

$$
\begin{aligned}
& M_{k-1,+}-m_{k,-} \leq c_{25}(k)\left\{M_{k-1,+}-M_{k,+}\right\}, \\
& M_{k,-}-m_{k-1,+} \leq c_{25}(k)\left\{m_{k,+}-m_{k-1,+}\right\} .
\end{aligned}
$$


By adding these two inequalities we have

$$
\begin{aligned}
M_{k,+}-m_{k,+} & \leq\left(1-\frac{1}{c_{25}(k)}\right)\left(M_{k-1,+}-m_{k-1,+}\right) \\
& \leq\left\{\prod_{l=2}^{k}\left(1-\frac{1}{c_{25}(l)}\right)\right\}\left(M_{1,+}-m_{1,+}\right) .
\end{aligned}
$$

Since $x \in \mathrm{R}_{0}$, it is not difficult to see $1 \leq \sup _{k} c_{25}(k)<\infty$, which implies

$$
\lim _{k \rightarrow \infty} \prod_{l=2}^{k}\left(1-\frac{1}{c_{25}(l)}\right)=0 .
$$

Hence, we obtain the claim.

Proof of Theorem 3.4. Part (1) follows from Lemma 7.1 and the Markov property.

The upper bound in (3.7) is immediate from (2.12).

To obtain the lower bound in (3.7), we see from the upper bound and $\lim \sup _{n \rightarrow \infty} N^{\alpha n} \mu\left(\overline{\mathbf{c}}_{n}\right)<\infty$ in (3.6) that

$$
c_{26} t^{-\alpha / 2} \leq \sup _{y \in \mathbf{c}_{n}\left(N^{-2} / 2\right)} p(t, y, x) \quad \text { for all } 0<t<c_{27}
$$

for some positive constants $c_{26}$ and $c_{27}$. By applying (6.4) for $\mathbf{c}_{n}\left(N^{-2} / 2\right)$ we obtain (3.7) but for restricted $t<c_{27}$ from (7.1). We used here the facts that the constant $c_{25}$ is independent of $n$ and that the time intervals $I_{n, \pm}$ can be taken in such a way that they cover $\left(0, c_{27}\right]$ because $x \in \mathrm{R}_{\alpha}$.

Since $p(t, x, x)$ is decreasing in $t$ and $p(1, x, x)$ is positive, we obtain (3.7) for all $0<t<1$, which completes the proof of (2).

Proof of Theorem 3.5. We observe that, by (3.1), $p$ has the self-similarity

$$
p(t, x, y)=N^{-\alpha k} p\left(t N^{-2 k}, x N^{-k}, y N^{-k}\right) .
$$

Hence, (1) follows from the self-similarity of $p(t, x, y)$ and the fact that $p$ is continuous at $(t, 0,0)$ for all $t$, which follows from Lemmas 3.3 and 7.1.

We remark that

$$
p(t, x, x)=\int p\left(\frac{1}{2}, x, y\right) p\left(t-\frac{1}{2}, y, x\right) d \mu(d y) .
$$

By combining this with and the fact that $p(t, y, x)$ is continuous at $(t, y, x)=$ $(1,0,0)$, we see that (3.9) follows from (3.8).

Part (3) follows from the fact that $\mu$ does not satisfies the doubling condition (see $[9,14,15])$. 


\section{Appendix A. Proof of Lemma 5.7}

Proof of Lemma 5.7. Without loss of generality we can assume $f \geq 0$. Let $M=$ $\mu(\mathcal{O}) / 2$ and $T=\inf \left\{t ; \mu\left(V_{t}\right)<M\right\}$, where $V_{t}=\{x ; f(x)>t\}$. We can assume

$$
\mu(\{f=T\})=0 .
$$

Otherwise, we can take a sequence $T_{n}$ satisfying $T_{n}>0, \lim T_{n}=T$ and (A.1) for $T_{n}$. By Minkowski's inequality we have

$$
\begin{aligned}
\|f\|_{L^{2 \mathbf{b}}(\mathcal{O}, \mu)} & =\left\{\int_{\mathcal{O} \backslash V_{T}} f^{2 \mathbf{b}} d \mu+\int_{V_{T}}\{(f-T)+T\}^{2 \mathbf{b}} d \mu\right\}^{1 / 2 \mathbf{b}} \\
& \leq\left\|f \cdot 1_{\mathcal{O} \backslash V_{T}}\right\|_{L^{2 \mathbf{b}}(\mathcal{O}, \mu)}+\left\|(f-T) \cdot 1_{V_{T}}\right\|_{L^{2 \mathbf{b}}(\mathcal{O}, \mu)}+T \mu\left(V_{T}\right)^{1 / 2 \mathbf{b}} .
\end{aligned}
$$

Here the function $1_{A}$ denotes the characteristic function of a set $A$. We estimate the three terms in the right-hand side of (A.2).

- The first term. By Hölder's inequality we see that

$$
\left\|f \cdot 1_{\mathcal{O} \backslash V_{T}}\right\|_{L^{2 \mathbf{b}}(\mathcal{O}, \mu)} \leq\left\|f \cdot 1_{\mathcal{O} \backslash V_{T}}\right\|_{L^{\mathbf{c}}(\mathcal{O}, \mu)}^{1 / \mathbf{b}} \cdot\left\|f \cdot 1_{\mathcal{O} \backslash V_{T}}\right\|_{L^{2}(\mathcal{O}, \mu)}^{1-1 / \mathbf{b}} .
$$

In addition, we have

$$
\begin{aligned}
\left\|f \cdot 1_{\mathcal{O} \backslash V_{T}}\right\|_{L^{\mathbf{c}}(\mathcal{O}, \mu)}^{\mathbf{c}} & \leq T^{\mathbf{c}-2} \cdot\left\|f \cdot 1_{\mathcal{O} \backslash V_{T}}\right\|_{L^{2}(\mathcal{O}, \mu)}^{2} \\
& \leq M^{-(\mathbf{c}-2) / 2} \cdot\left\|f \cdot 1_{V_{T}}\right\|_{L^{2}(\mathcal{O}, \mu)}^{\mathbf{c}-2} \cdot\left\|f \cdot 1_{\mathcal{O} \backslash V_{T}}\right\|_{L^{2}(\mathcal{O}, \mu)}^{2} \\
& \leq M^{-(\mathbf{c}-2) / 2} \cdot\|f\|_{L^{2}(\mathcal{O}, \mu)}^{\mathbf{c}} .
\end{aligned}
$$

Combining this with (A.3) and using $(\mathbf{c}-2) / 2 \mathbf{c}=1 / \mathbf{a}$ we obtain

$$
\left\|f \cdot 1_{\mathcal{O} \backslash V_{T}}\right\|_{L^{2} \mathbf{b}(\mathcal{O}, \mu)} \leq\left(M^{-1 / \mathbf{a}}\|f\|_{L^{2}(\mathcal{O}, \mu)}\right)^{1 / \mathbf{b}}\|f\|_{L^{2}(\mathcal{O}, \mu)}^{1-1 / \mathbf{b}} .
$$

- The second term. By Hölder's inequality again we see that

$$
\begin{aligned}
\left\|(f-T) \cdot 1_{V_{T}}\right\|_{L^{2 \mathbf{b}}(\mathcal{O}, \mu)} & \leq\left\|(f-T) \cdot 1_{V_{T}}\right\|_{L^{\mathbf{c}}(\mathcal{O}, \mu)}^{1 / \mathbf{b}}\left\|(f-T) \cdot 1_{V_{T}}\right\|_{L^{2}(\mathcal{O}, \mu)}^{1-1 / \mathbf{b}} \\
& \leq\left\|(f-T) \cdot 1_{V_{T}}\right\|_{L^{\mathbf{c}}(\mathcal{O}, \mu)}^{1 / \mathbf{b}}\|f\|_{L^{2}(\mathcal{O}, \mu)}^{1-1 / \mathbf{b}} .
\end{aligned}
$$

Applying (5.28) to $(f-T) \cdot 1_{V_{T}}$, we have

$$
\begin{aligned}
\left\|(f-T) \cdot 1_{V_{T}}\right\|_{L^{\mathbf{c}}(\mathcal{O}, \mu)} & \leq c_{20}(\mu, \mathbf{a})\left\|\nabla(f-T) \cdot 1_{V_{T}}\right\|_{L^{2}(\mathcal{O}, \mu)} \\
& \leq c_{20}(\mu, \mathbf{a})\|\nabla f\|_{L^{2}(\mathcal{O}, \mu)} .
\end{aligned}
$$


Although $(f-T) \cdot 1_{V_{T}}$ is not in $C_{0}^{\infty}\left(\mathbb{R}^{d}\right)$, we can still apply Lemma 5.5 by approximation of elements in $C_{0}^{\infty}\left(\mathbb{R}^{d}\right)$. For the second inequality we have used

$$
\nabla\left((f-T) \cdot 1_{V_{T}}\right)(x)=1_{V_{T}}(x) \cdot \nabla f(x) \text { for } \mu \text {-almost every } x,
$$

which follows from (A.1). Combining these inequalities yields

$$
\left\|(f-T) \cdot 1_{V_{T}}\right\|_{L^{2 \mathbf{b}}(\mathcal{O}, \mu)} \leq c_{20}(\mu, \mathbf{a})^{1-1 / \mathbf{b}}\|\nabla f\|_{L^{2}(\mathcal{O}, \mu)}^{1 / \mathbf{b}}\|f\|_{L^{2}(\mathcal{O}, \mu)}^{1-1 / \mathbf{b}} .
$$

- The third term. Since $f \geq T$ on $V_{T}$ and $\mu\left(V_{T}\right) \geq M$, we have

$$
T^{2 \mathbf{b}} \mu\left(V_{T}\right) \leq\left(M^{-1 / \mathbf{a}}\|f\|_{L^{2}(\mathcal{O}, \mu)}\right)^{1 / \mathbf{b}}\|f\|_{L^{2}(\mathcal{O}, \mu)}^{1-1 / \mathbf{b}} .
$$

Combining (A.4), (A.5) and (A.6) with (A.2) we obtain (5.33).

\section{REFERENCES}

[1] D. G. Aronson and J. Serrin. Local behavior of solutions of quasilinear parabolic equations. Arch. Ration. Mech. Anal. 25 (1967), 81-122.

[2] M. Barlow and R. Bass. The construction of Brownian motion on the Sierpiński carpet. Ann. Inst. H. Poincaré Probab. Statist. 25 (1989), 225-257.

[3] M. Barlow and R. Bass. Brownian motion and harmonic analysis on Sierpiński carpets. Canad. J. Math. 51 (1999), 673-744.

[4] E. A. Carlen, S. Kusuoka and D. W. Stroock. Upper bounds for symmetric Markov transition functions. Ann. Inst. H. Poincaré Probab. Statist. 2 (1987), 245-287.

[5] I. Chavel. Eigenvalues in Riemannian Geometry. Academic Press, New York, 1984.

[6] M. Fukushima. On a stochastic calculus related to Dirichlet forms and distorted Brownian motions. New stochastic methods in physics. Phys. Rep. 77(3) (1981), 255-262.

[7] M. Fukushima. Distorted Brownian motions and BV functions. Trends in Probability and Related Analysis (Taipei, 1996). World Scientific, Singapore, 1997, pp. 143-150.

[8] M. Fukushima, Y. Oshima and M. Takeda. Dirichlet Forms and Symmetric Markov Processes. Walter de Gruyter, Berlin, 1994.

[9] A. A. Grigor'yan. Heat equation on non-compact Riemannian manifold. Math. USSR Sb. 72 (1992), 47-77.

[10] A. N. Kolmogoroff. Über die analytischen methoden in der Wahrcsheinlichkeitsrechnung. Math. Ann. 104 (1931), 415-458.

[11] H. Osada. A family of diffusion processes on Sierpiński carpets. Probab. Theory Related Fields 119 (2001), 275-310.

[12] H. Osada. Harnack inequalities for exotic Brownian motions. Kyushu J. Math. 56 (2002), 363-380.

[13] H. Osada. Singular time changes of diffusions on Sierpiński carpets. Stochastic Process. Appl. 116 (2006), 675-689.

[14] L. Saloff-Coste. A note on Poincaré, Sobolev, and Harnack inequalities. Duke Math. J. 65 (1992), $27-32$.

[15] K. T. Sturm. Analysis on local Dirichet spaces III. The parabolic Harnack inequality. J. Math. Pures Appl. (9) 75 (1996), 273-297. 
Exotic Brownian motions

Faculty of Mathematics

Kyushu University 33

Fukuoka 812-8581

Japan

(E-mail: osada@math.kyushu-u.ac.jp) 